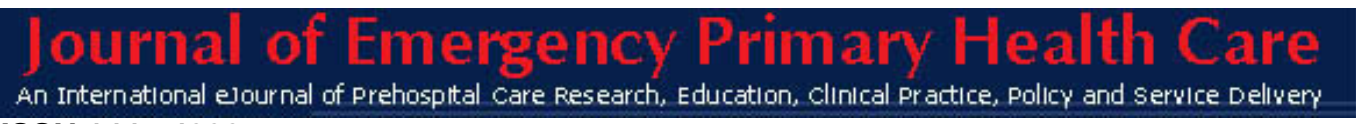

ISSN 1447-4999

\title{
CD-ROM REVIEW
}

\section{Brett Williams}

Lecturer

Monash University Centre for Ambulance and Paramedic Studies

"eACLS"

\author{
American College of Emergency Physicians \& National Safety Council. \\ February 2004. ISBN 0-7637-3229-X \\ Recommended retail price $\$ 159.50$. AUD
}

This CD Rom is an interactive case-based program developed earlier in the year by the American College of Emergency Physicians and the National Safety Council. It has two tracks of care: Hospital and Prehospital. Both of which comprise of ten individual cases:

Acute Coronary Syndrome

Automatic External Defibrillation

Asystole

Bradycardia

Narrow Complex Tachycardia

Pulseless Electrical Activity

Respiratory Arrest

Stroke

Ventricular Fibrillation

Wide Complex Tachycardia

This is an innovative attempt at delivering an educational paradigm such as case-based learning into the virtual e-learning environment. It has tremendous potential. The CD is easy to navigate and has clear and succinct voice over instructions that 'walk' you through the main features and keys required during your learning.

Each scenario has learning objectives that in most cases are adequate and prepare the learner for their journey. Each case has five sections that must be completed sequentially:

Your Patient

Assessment

Treatment

Interactivities

Simulations 
The first three sections provide refresher information relative to a patient with a particular medical problem (e.g. AMI). The fourth part, "Interactivities" tests the general understanding of the refresher content. This section includes electrocardiograms (ECG's), clinical manifestations and specific pharmacological multiple-choice questions. Importantly, feedback is provided when an error in this section occurs, this provides the user with a clear indication where they went wrong. The final section "Simulations" tests the ability to apply knowledge of this topic to a patient experiencing a similar emergency. The section incorporates both video and photographs in a casebased series in 'real-life' and 'real-time' which allows the user to develop a more realistic approach. Again, this section will allow individuals to make mistakes and provide enough information to finally obtain the correct assessment or treatment. The history component is very useful in this section as it allows maintenance of a running log of previous actions.

As an educator, the biggest positive from the simulation section was the repetitive need for users to be sequential in primary approaches and in Advanced Life Support (ALS) management. For example, cases could only be continued or completed when the ABC's and other priorities had been addressed.

The resources section has more than enough information to successfully complete all ten cases. In other words, users do not require any other texts or aids to complete this program. They include sixteen different ECG's, Electrical Therapy and seventeen Advanced Cardiac Life Support (ACLS) medications.

The opportunity also exists to complete the final test after all ten cases have been accomplished. This is secured and must achieve a minimum score of $84 \%$ to pass the course and attain your course completion card.

Whilst the program is American-based it provides a different approach to procedures and ACLS medications than in Australia. It reinforces the importance of developing a broad research scope to become familiar with International Standards. For example, it is motivating to use procainamide or vasopressin in the simulation despite not using it in local clinical protocols or guidelines. It is also satisfying to see certain doses, such as aspirin have been altered following large studies such as ISIS, GISIS and GUSTO.

So, what areas could be improved?

Perhaps the ECG grid paper could be clearer. I imagine for the novice it would be potentially difficult to interpret certain rhythms because of a lack of clarity. Eight of the ten cases were cardiac-orientated, perhaps a greater variety of cases could have been utilised. Possibly further medical, trauma or paediatric cases could be developed. Some statements are not adequately referenced, such as "LMA's and Combitubes are almost as effective as Endotracheal Tubes".

Who is best suited for this program? I think any educator who is teaching under a case-based or problem-based format would find this an important 
teaching adjunct. It provides 'real-life' simulations that reinforce the importance of primary care approaches and sequential ALS clinical problem solving skills. Students that would be most suited to this program would range between post six months training (pre \& post employment paramedics) up to pre-Intensive Care Paramedics.

I believe there is also scope for qualified Intensive Care Paramedics to use this program as a refresher tool. Ambulance organisations could also use the program as part of their 'in-service' training schedule or to maintain quality assurance amongst their staff.

To successfully run this program you will require the following minimum system requirements:

\begin{tabular}{|l|l|}
\hline \multicolumn{1}{|c|}{ Windows (98/2000/NT/XP) } \\
\begin{tabular}{|l|l|}
$128 \mathrm{MB}$ RAM (256 MB RAM of higher \\
recommended)
\end{tabular} & $\begin{array}{l}68030 \text { ('040 or PowerMac running at } \\
120 \mathrm{MHz} \text { or more recommended) } \\
32 \mathrm{MB} \text { RAM (64 MB RAM or higher } \\
\text { recommended) } \\
640 \times 480 \text { screen resolution or higher, } \\
\text { hi-colour mode } \\
200 \mathrm{MB} \text { free hard drive } \\
\text { higher } \mathrm{CD} \text { Rom drive (4 x speed or } \\
120 \mathrm{MB} \text { of available hard drive space }\end{array}$ \\
\hline
\end{tabular}

